\title{
SOME STABILITY RESULTS FOR THE COMPLEX GINZBURG-LANDAU EQUATION
}

\author{
SIMÃO CORREIA AND MÁRIO FIGUEIRA
}

\begin{abstract}
Using some classical methods of dynamical systems, stability results and asymptotic decay of strong solutions for the complex Ginzburg-Landau equation (CGL),

$$
\partial_{t} u=(a+i \alpha) \Delta u-(b+i \beta)|u|^{\sigma} u+k u, t>0, x \in \Omega,
$$

with $a>0, \alpha, b, \beta, k \in \mathbb{R}$, are obtained. Moreover, we show the existence of bound-states under certain conditions on the parameters and on the domain. We conclude with the proof of asymptotic stability of these bound-states when $\Omega=\mathbb{R}$ and $-k$ large enough.
\end{abstract}

Keywords: complex Ginzburg-Landau; stability; bound-states.

AMS Subject Classification 2010: 35Q56, 35B10, 35B35.

\section{INTRODUCTION AND MAIN RESULTS}

This paper is concerned with the study of the stability of some equilibrium solutions of the Cauchy problem for the complex Ginzburg-Landau equation

$$
\left\{\begin{array}{l}
\partial_{t} u=(a+i \alpha) \Delta u-(b+i \beta)|u|^{\sigma} u+k u, \quad t>0, x \in \Omega \\
u(t, x)=0, \quad x \in \partial \Omega, t \geq 0 \\
u(0, x)=u_{0}(x)
\end{array}\right.
$$

where $a>0, \alpha, b, \beta, k \in \mathbb{R}$ and $\Omega$ is assumed to be a domain in $\mathbb{R}^{N}$ of class $C^{2}$ with $\partial \Omega$ bounded. We also analyze the asymptotic behaviour of the global solutions of (CGL). Local and global existence and uniqueness of solutions of (CGL) are widely studied under several assumptions on the parameters since the seminal paper [11]; see also [6],[7],[13] and the references therein. On the other hand, there are not many results concerning the blow-up of the solutions of (CGL): we refer e.g. [2] and [12]. Furthermore, concerning the existence of standing wave solutions, some partial results were obtained in the case of a bounded connected domain; cf.[3] and [5]. The latter also includes a result concerning the whole space $\Omega=\mathbb{R}^{N}$.

The linear operator of (CGL), $-A=(a+i b) \Delta, a>0, b \in \mathbb{R}$, with domain, $D(A)=H^{2}(\Omega) \cap H_{0}^{1}(\Omega)$, generates an analytic semi-group (see [14]): writing $A_{\varepsilon}=A+\varepsilon I, \varepsilon>0$, we have $\Re\left(A_{\varepsilon} u, u\right)_{L^{2}}=a\|\nabla u\|_{L^{2}}^{2}+\varepsilon\|u\|_{L^{2}}^{2} \geq$ $c_{0}\|u\|_{H^{1}}^{2}, c_{0}=\min \{a, \varepsilon\}$ and $\left|\Im\left(A_{\varepsilon} u, u\right)_{L^{2}}\right| \leq|b|\|u\|_{H^{1}}^{2}$. Then, the resolvent set

$$
\rho\left(A_{\varepsilon}\right) \supset\left\{\lambda:|\arg \lambda|>\theta_{\varepsilon}\right\}, \theta_{\varepsilon}=\arctan (|b| / \varepsilon)<\pi / 2
$$


and if we choose $\varepsilon$ small enough, we conclude that $\rho(A) \supset\{\lambda: \Re \lambda<0\}$. We see also easily that $e^{-A z}=e^{-A_{a} z} e^{a z}$ is an analytic semi-group in the sector $\{z:|\arg z|<\omega\}, \omega=\pi / 2-\arctan (|b| / a)$.

Let introduce now the following definition:

Definition 1.1. A function $u(\cdot) \in C\left([0, T) ; L^{2}(\Omega)\right), T>0$, is called a strong solution of $(\mathrm{CGL})$ if $u(t) \in D(A), \frac{d u}{d t}(t)$ exists for $t \in(0, T), u(0)=$ $u_{0}$ and the differential equation in (CGL) is satisfied for $t \in(0, T)$.

Since $f(u)=-(b+i \beta)|u|^{\sigma} u+k u$ is locally Lipschitz in $H^{1}(\Omega)$ with values in $L^{2}(\Omega)$, for $\sigma \leq \frac{2}{N-2}$ if $N>2$ and for any $\sigma>0$ if $N=1,2$, then there exists $T=T\left(u_{0}\right)>0$ such that the problem (CGL) has a unique solution on $\left[0, T_{0}\right)$, and this solution depends continuously of the initial data (see [10], pag. 54 and 62). To obtain a global solution, multiply the equation in (CGL) by $\bar{u},-\Delta \bar{u}$ and $|u|^{\sigma} \bar{u}$, integrate on $\Omega$ and take the real part. One obtains

$$
\begin{gathered}
\frac{1}{2} \frac{d}{d t}\|u\|_{L^{2}}^{2}=-a\|\nabla u\|_{L^{2}}^{2}-b\|u\|_{L^{\sigma+2}}^{\sigma+2}+k\|u\|_{L^{2}} \\
\frac{1}{2} \frac{d}{d t}\|\nabla u\|_{L^{2}}^{2}=-a\|\Delta u\|_{L^{2}}^{2}+b \Re \int_{\Omega} \Delta \bar{u}|u|^{\sigma} u d x \\
-\beta \Im \int_{\Omega} \Delta \bar{u}|u|^{\sigma} u d x+k\|\nabla u\|_{L^{2}}^{2} \\
\frac{1}{\sigma+2} \frac{d}{d t}\|u\|_{L^{\sigma+2}}^{\sigma+2}=a \Re \int_{\Omega} \Delta u|u|^{\sigma} \bar{u} d x-\alpha \Im \int_{\Omega} \Delta u|u|^{\sigma} \bar{u} d x \\
-b\|u\|_{L^{2 \sigma+2}}^{2 \sigma+2}+k\|u\|_{L^{\sigma+2}}^{\sigma+2}
\end{gathered}
$$

Next, if we multiply (1.3) by $\beta / \alpha$ (with $\alpha \neq 0$ ) and add to $(1.1)+(1.2)$, one obtains

$$
\begin{aligned}
\frac{d}{d t} & {\left[\frac{1}{2}\|u\|_{H^{1}}^{2}+\frac{\beta}{\alpha(\sigma+2)}\|u\|_{L^{\sigma+2}}^{\sigma+2}\right]=k\left[\|u\|_{H^{1}}^{2}+\frac{\beta}{\alpha}\|u\|_{L^{\sigma+2}}^{\sigma+2}\right]-a\|\nabla u\|_{L^{2}}^{2} } \\
& -b\|u\|_{L^{\sigma+2}}^{\sigma+2}-a\|\Delta u\|_{L^{2}}^{2}-\frac{\beta b}{\alpha}\|u\|_{L^{2 \sigma+2}}^{2 \sigma+2}+\left(b+\frac{a \beta}{\alpha}\right) \Re \int_{\Omega} \Delta u|u|^{\sigma} \bar{u} d x
\end{aligned}
$$

Since $\Re \int_{\Omega} \Delta u|u|^{\sigma} \bar{u} d x \leq 0$, it is now clear that, if $b \geq 0$ and $\alpha \beta \geq 0$ then $\|u(t)\|_{H^{1}}$ is locally bounded and we can state:

Proposition 1.2. Let $\Omega$ be a domain in $\mathbb{R}^{N}$ of class $C^{2}$ with $\partial \Omega$ bounded. Assume $0<\sigma \leq \frac{2}{N-2}$ if $N>2$ and $0<\sigma$ if $N=1,2$. Then, for any $u_{0} \in H_{0}^{1}(\Omega)$, there exists $T=T\left(u_{0}\right)>0$ such that (CGL) has a unique strong solution on $[0, T)$ and this solution depends continuously of the initial data. Moreover, if $b+\alpha \beta / a \geq 0$, the solution is global. 
Under the conditions of the Proposition 1.2, denote by $S(t)$ the dynamical system associated to (CGL) such that $S(t) u_{0} \equiv u\left(t ; u_{0}\right), t \geq 0$, represents the solution of (CGL) with initial data $u(0)=u_{0} \in H_{0}^{1}(\Omega)$, and let recall the following classical definition:

Definition 1.3. We say that $u_{0} \in H_{0}^{1}(\Omega)$ is stable if for any $\delta>0$ there exists $\varepsilon>0$ such that

$$
v \in H_{0}^{1}(\Omega),\left\|u_{0}-v\right\|_{H^{1}}<\varepsilon \Rightarrow \sup _{t \geq 0}\left\|S(t) u_{0}-S(t) v\right\|_{H^{1}}<\delta
$$

In addition, we say that $u_{0}$ is asymptotically stable if $u_{0}$ is stable and there exists $\eta>0$ such that $\lim _{t \rightarrow \infty}\left\|S(t) u_{0}-S(t) y\right\|_{H^{1}}=0$ for all $y \in$ $H_{0}^{1}(\Omega),\left\|u_{0}-y\right\|_{H^{1}}<\eta$.

The following results concern the stability of the equilibrium solution $u \equiv 0$ and the asymptotic decay of the global solutions of (CGL) depending on the coefficient for the driving term $k$.

Theorem 1.4 (Stability of the zero solution for small $k$ ). Assume the hypothesis of the Proposition 1.2.

1. $L^{p}$ stability:

If

$$
k \leq 0 \quad \text { and } \quad \frac{|\alpha|}{a} \leq \frac{2}{p-2}
$$

the equilibrium point 0 is $L^{p}$-stable for $2 \leq p \leq \frac{2 N}{N-2}$, if $N>2,2 \leq p<$ $\infty$ if $N=1,2$.

In addition, if $k<0$

$$
\|u(t, x)\|_{L^{p}} \rightarrow 0 \text { as } t \rightarrow \infty, \quad \text { for all } u_{0} \in H_{0}^{1}(\Omega) .
$$

In the particular case $p=2$, if $\Omega$ is a bounded domain, $k>0$ and $\frac{k}{a}<\left(\frac{1}{\omega_{N}}|\Omega|\right)^{-2 / N}$, where $\omega_{N}$ represents the volume of the unit ball in $\mathbb{R}^{N}$ and $|\Omega|$ the volume of $\Omega$, then $\|u(t, x)\|_{L^{2}} \rightarrow 0$ as $t \rightarrow \infty$, for all $u_{0} \in H_{0}^{1}(\Omega)$.

2. $H^{1}$ stability:

Let $\Omega$ a bounded domain and assume $\alpha / a=\beta / b$. Then 0 is asymptotically stable in $H^{1}$ if

$$
k \leq \frac{a}{2}\left(\frac{1}{\omega_{N}}|\Omega|\right)^{-2 / N}
$$

In addition,

$$
\|u(t, x)\|_{H^{1}} \rightarrow 0 \text { as } t \rightarrow \infty, \quad \text { for all } u_{0} \in H_{0}^{1}(\Omega) .
$$

Theorem 1.5 (Instability of the zero solution for large $k$ ). Let $\Omega \subset \mathbb{R}^{N}$ be a bounded domain of class $C^{2}$ and denote by $\lambda_{n}, n=1,2, \ldots$ the eigenvalues of $-\Delta$ ranked in ascending order. Consider the (CGL) problem with $0<\sigma \leq$ $\frac{2}{N-2}$ if $N>2$ and $0<\sigma$ if $N=1,2$ and assume $b<0, \alpha, \beta \in \mathbb{R}, \alpha / a=\beta / b$ 
and $k>0$ such that $k / a \in\left(\lambda_{n}, \lambda_{n+1}\right)$ for some $n \in \mathbb{N}$. Then, the equilibrium solution $u \equiv 0$ is unstable.

As we have noted before, the existence of standing waves for the complex Ginzbourg-Landau equation remains a largely open problem. Before we proceed, we rewrite the complex Ginzburg-Landau equation in its trigonometric form, following the notations of [3] and [5]:

$$
u_{t}=e^{i \theta} \Delta u+e^{i \gamma}|u|^{\sigma} u+k u
$$

where $-\pi / 2<\theta<\pi / 2,-\pi<\gamma \leq \pi, k \in \mathbb{R}, u=u(t, x), x \in \mathbb{R}, t>0$. We then look for solutions of (CGL*) in the form $u=e^{i \omega t} \phi(x)$, where $\phi \in H^{1}(\mathbb{R})$ is a solution of the elliptic equation

$$
i \omega \phi=e^{i \theta} \Delta \phi+e^{i \gamma}|\phi|^{\sigma} \phi+k \phi, \quad \phi \in H_{0}^{1}(\Omega) .
$$

To stress the difficulty in finding bound-states, we start with the following simple non-existence result:

Proposition 1.6. Given $\Omega$ an open subset of $\mathbb{R}^{d}$, suppose that $\phi \in H_{0}^{1}(\Omega)$ is a solution of

$$
i \omega \phi=e^{i \theta} \Delta \phi+e^{i \gamma}|\phi|^{\sigma} \phi, \quad \omega>0,0 \leq \gamma \leq \theta \leq \pi / 2, \gamma \neq \pi / 2 .
$$

Then $\phi \equiv 0$. The same conclusion is valid if $\omega=0$ and $\theta \neq \gamma$.

Remark 1.7. Notice that (B-S) may always be reduced to $k=0$ : it suffices to multiply the equation by $(\omega+i k) /\left(\omega^{2}+k^{2}\right)$.

As a consequence of the non-existence result, one can argue that a direct perturbative argument around the Schrdinger ground-state is impossible. In fact, the Schrdinger case corresponds to $\theta=\gamma=\pi / 2$ and $\omega=1$, which lies in the frontier of the region of non-existence. If one could apply a direct perturbative argument (that is, without having a dependence between $\gamma$ and $\theta$ ), one would find an open region of existence around $\theta=\gamma=\pi / 2$, which is impossible.

Our contribution to the problem of existence of bound-states for (CGL*) is twofold: the first result concerns the existence on bounded domains, for certain values of $k$; the second focuses on the case $\Omega=\mathbb{R}$.

Theorem 1.8. Suppose that $\Omega$ is a bounded, connected, open subset of $\mathbb{R}^{d}$ such that the Laplace-Dirichlet operator over $\Omega$ has a simple eigenvalue $\lambda$. Fix $0 \leq \theta, \gamma \leq \pi / 2$ and $\gamma \neq \pi / 2$. There exists $\epsilon>0$ such that, for any $k$ with $0<\lambda \cos \theta-k<\epsilon$, there exist $\omega>0$ and a solution $\phi \in H_{0}^{1}(\Omega)$ of (B-S)

Remark 1.9. The above result is quite similar to that of [3]: therein, it is proven that, over open bounded connected subsets of $\mathbb{R}^{d}$, the equation

$$
i \omega u=e^{i \theta} \Delta u+e^{i \gamma}|u|^{\sigma} u+k u
$$


has a solution $(\omega, u) \in \mathbb{R} \times H_{0}^{1}(\Omega)$ if $\sigma$ is sufficiently small and $(\lambda \cos \theta-$ $k) \cos \gamma>0$. The goal of our result is to trade the freedom in $k$ for the freedom in $\sigma$.

Theorem 1.10. Consider $\Omega=\mathbb{R}$.

1. If $\omega^{2}+k^{2} \neq 0$ and $\arg (k-i \omega) \neq \theta$, then equation (B-S) has at most one solution, up to gauge rotations and translations.

2. Fix $-\pi / 2<\theta<\pi / 2$ and $\omega, k \in \mathbb{R}$ such that $\omega \cos \theta+k \sin \theta \neq 0$. Define

$$
d=\frac{k \cos \theta-\omega \sin \theta+\sqrt{\omega^{2}+k^{2}}}{\omega \cos \theta+k \sin \theta}
$$

and let $\gamma \in(-\pi, \pi]$ be the unique solution of

$$
\tan (\gamma-\theta)=\frac{d(\sigma+4)}{\sigma+2-2 d^{2}}, \quad d \sin (\gamma-\theta)+\cos (\gamma-\theta)>0 .
$$

Then the complex Ginzburg-Landau equation admits a bound-state of the form

$$
\phi=\psi \exp (i d \ln \psi),
$$

where $\psi$ is the bound-state for the nonlinear Schrdinger equation, up to scaling and scalar multiplication.

Remark 1.11. We observe that the conditions $\omega^{2}+k^{2} \neq 0$ and $\arg (k-i \omega) \neq$ $\theta$ imply $\omega \cos \theta+k \sin \theta \neq 0$.

Remark 1.12. In the particular case $\omega=1$ and $k=0$, the condition (1.5) reduces to

$$
\frac{\cos \gamma}{\sin (\gamma-\theta)}=\frac{\sigma}{\sigma+4}
$$

In particular, $\gamma>\theta$, which agrees with the nonexistence result. Moreover, for $\gamma \leq \pi / 4$, the above condition is never verified:

$$
\frac{\cos \gamma}{\sin (\gamma-\theta)}>\frac{\cos \gamma}{\sin \gamma} \geq 1>\frac{\sigma}{\sigma+4} .
$$

Finally, we present a stability result for bound-states with large $-k$ :

Theorem 1.13. Fix $\omega=1$ and $0<\theta<\pi / 2$. Then, for $-k$ sufficiently large, the bound-state $\phi$ built in Theorem 1.10 is asymptotically stable.

\section{Proof of the Theorem 1.4}

Let $S(t)$ be a dynamical system on a Banach space $H$ and recall that a Lyapunov function is a continuous function $W: H \rightarrow \mathbb{R}$ such that

$$
\dot{W}(u):=\limsup _{t \rightarrow 0^{+}} \frac{1}{t}[W(S(t) u)-W(u)] \leq 0
$$

for all $u \in H$. The next lemma is mainly proved in [9]. 
Lemma 2.1. Let $S(t)$ be a dynamical system on a Banach space $(D,\|\|)$. Let $E$ a normed space such that $D \hookrightarrow E$ and $W$ a Lyapunov function on $D$ such that

$$
W\left(u_{0}\right) \geq k_{1}\left\|u_{0}\right\|_{E}, k_{1}>0, u_{0} \in D .
$$

Then, the equilibrium point 0 is \|\|$_{E}$ - stable in the sense that

$$
u_{0} \in D,\left\|u_{0}\right\| \rightarrow 0 \Rightarrow\left\|S(t) u_{0}\right\|_{E} \rightarrow 0,
$$

uniformly in $t \geq 0$.

Assume in addition that

$$
\dot{W}\left(u_{0}\right) \leq-k_{2}\left\|u_{0}\right\|_{E}, k_{2}>0, u_{0} \in D .
$$

Then, $\lim _{t \rightarrow \infty}\left\|S(t) u_{0}\right\|_{E}=0$ for any $u_{0} \in D$.

Proof of Theorem 1.4. 1. Let denote by $S(t)$ the dynamical system associated to (CGL), where $S(t) u_{0} \equiv u\left(t, u_{0}\right)$ represents the unique global solution of (CGL) under the hypothesis of the Proposition (1.2) and define

$$
W_{p}(u)=\int_{\Omega}|u(x)|^{p} d x
$$

with $2 \leq p \leq \frac{2 N}{N-2}$ if $N>2,2 \leq p<\infty$ if $N=1,2$ and $u=u\left(t, u_{0}\right)$. It is clear that $W_{p}: H_{0}^{1}(\Omega) \rightarrow \mathbb{R}$, is a continuous functional and, from $\dot{W}_{p}(u)=\nabla W(u) \cdot \frac{d}{d t} u(t)$, we get

$$
\begin{aligned}
\dot{W}_{p}(u)= & p \Re \int_{\Omega}|u|^{p-2} \bar{u}\left\{(a+i \alpha) \Delta u-(b+i \beta)|u|^{\sigma} u+k u\right\} d x \\
\leq & p k \int_{\Omega}|u|^{p} d x-a p \int_{\Omega}|u|^{p-2}|\nabla u|^{2} d x \\
& -p b \int_{\Omega}|u|^{p}|u|^{\sigma} d x+p \alpha \Im \int_{\Omega} \nabla\left(|u|^{p-2}\right) \bar{u} \nabla u d x .
\end{aligned}
$$

Since

$$
\nabla|u|^{p-2}=\frac{p-2}{2}|u|^{p-4}(u \nabla \bar{u}+\bar{u} \nabla u)
$$

we obtain

$$
\left|p \alpha \Im \int_{\Omega} \nabla\left(|u|^{p-2}\right) \bar{u} \nabla u d x\right| \leq p|\alpha| \frac{p-2}{2} \int_{\Omega}|u|^{p-2}|\nabla u|^{2} d x .
$$

Hence, if $\alpha \frac{p-2}{2} \leq a$ and $k \leq 0$ we derive that $\dot{W}_{p}(u) \leq p k\|u\|_{L^{p}}^{p}$ and the conclusion follows from the Lemma 2.1. If $p=2$ and $\Omega$ is bounded, the above estimation reduces to

$$
\dot{W}_{2}(u)=-2 a \int_{\Omega}|\nabla u|^{2} d x-2 b \int_{\Omega}|u|^{\sigma+2} d x+2 k \int_{\Omega}|u|^{2} d x
$$

and if $k\left(\frac{1}{\omega_{N}}|\Omega|\right)^{2 / N}<a$, by Poincaré inequality, the conclusion remains valid. 
2. We now consider $\Omega$ a bounded domain and we define a new functional:

$$
V(u):=\frac{a}{2} \int_{\Omega}|\nabla u|^{2} d x+\frac{b}{\sigma+2} \int_{\Omega}|u|^{\sigma+2} d x-\frac{k}{2} \int_{\Omega}|u|^{2} d x .
$$

$V$ is a continuous real function on $H_{0}^{1}(\Omega)$ and, if

$$
k \leq 0 \quad \text { or } \quad k>0, \frac{k}{a}<\left(\frac{1}{\omega_{N}}|\Omega|\right)^{2 / N}
$$

we have $V(u) \geq c\|u\|_{H^{1}(\Omega)}, c>0$. In addition, for any $u \in H_{0}^{1}(\Omega) \cap H^{2}(\Omega)$ and $h \in H_{0}^{1}(\Omega)$ we have $V(u+h)=V(u)+L \cdot h+o\left(\|h\|_{H^{1}}\right)$, where

$$
L \cdot h=-\Re \int_{\Omega}\left[a \Delta \bar{u}-b|u|^{\sigma} \bar{u}+k \bar{u}\right] h d x .
$$

Therefore, for all $u=u(t) \in H_{0}^{1}(\Omega) \cap H^{2}(\Omega)$ we get

$$
\begin{aligned}
\dot{V}(u)= & -\left.\int_{\Omega}|a \Delta u-b| u\right|^{\sigma} u+\left.k u\right|^{2} d x \\
& -\Re \int_{\Omega}\left(a \Delta u-b|u|^{\sigma} u\right) i\left(\alpha \Delta u-\beta|u|^{\sigma} u\right) d x \\
& -\Re \int_{\Omega} i k \bar{u}\left(\alpha \Delta u-\beta|u|^{\sigma} u\right) d x
\end{aligned}
$$

and, for $\frac{\alpha}{a}=\frac{\beta}{b}=c, c \in \mathbb{R}$, we obtain

$$
\dot{V}(u(t))=-\left.\int_{\Omega}|a \Delta u-b| u\right|^{\sigma} u+\left.k u\right|^{2} d x \leq 0, t>0 .
$$

Note that

$$
\frac{1}{t}\left[V\left(S(t) u_{0}\right)-V\left(u_{0}\right)\right]=\dot{V}\left(S\left(t^{*}\right) u_{0}\right)
$$

for some $0<t^{*}<t$ and so (2.4) is true for all $t \geq 0$. Hence, the functional $V$ is a Lyapunov function and we have the stability in $H_{0}^{1}(\Omega)$ of the equilibrium solution $u \equiv 0$.

We prove now the asymptotic stability. First, we remark that

$$
\begin{aligned}
-\dot{V}(u)= & a^{2} \int_{\Omega}|\Delta u|^{2} d x+b^{2} \int_{\Omega}|u|^{2 \sigma+2} d x+k^{2} \int_{\Omega}|u|^{2} d x \\
& -2 a b \Re \int_{\Omega} \Delta u|u|^{\sigma} \bar{u} d x+2 a k \Re \int_{\Omega} \Delta u \bar{u} d x \\
& -2 b k \Re \int_{\Omega}|u|^{\sigma+2} d x .
\end{aligned}
$$

The first, fourth, fifth and sixth terms in the right hand side can be estimate as follows :

$$
\int_{\Omega}|\nabla u|^{2} d x \leq\left|\int_{\Omega} \Delta u \bar{u} d x\right| \leq\left(\int_{\Omega}|\Delta u|^{2} d x\right)^{1 / 2}\left(\int_{\Omega}|u|^{2} d x\right)^{1 / 2}
$$


and by the Poincaré innequality,

$$
\left(\int_{\Omega}|\Delta u|^{2} d x\right)^{1 / 2} \geq\left(\frac{1}{\omega_{N}}|\Omega|\right)^{-1 / N}\left(\int_{\Omega}|\nabla u|^{2} d x\right)^{1 / 2}
$$

It follows that

$$
a^{2} \int_{\Omega}|\Delta u|^{2} d x \geq a^{2}\left(\frac{1}{\omega_{N}}|\Omega|\right)^{-2 / N} \int_{\Omega}|\nabla u|^{2} d x .
$$

Next

$$
\begin{aligned}
\Re \int_{\Omega} \Delta u|u|^{\sigma} \bar{u} d x= & -\int_{\Omega}|\nabla u|^{2}|u|^{\sigma} d x \\
& -\frac{\sigma}{2} \Re \int_{\Omega}|u|^{\sigma-2} \nabla u \cdot(\nabla u \bar{u}+u \nabla \bar{u}) \bar{u} d x \\
= & -\int_{\Omega}|\nabla u|^{2}|u|^{\sigma} d x-\frac{\sigma}{2} \int_{\Omega}|\nabla u|^{2}|u|^{\sigma} d x \\
& -\frac{\sigma}{2} \Re \int_{\Omega}|u|^{\sigma-2}(\nabla u \cdot \nabla u) \bar{u}^{2} d x \\
\leq & -\int_{\Omega}|\nabla u|^{2}|u|^{\sigma} d x
\end{aligned}
$$

and so

$$
-2 a b \Re \int_{\Omega} \Delta u|u|^{\sigma} \bar{u} d x \geq 2 a b \int_{\Omega}|\nabla u|^{2}|u|^{\sigma} d x .
$$

Also

$$
2 a k \Re \int_{\Omega} \Delta u \bar{u} d x=-2 a k \int_{\Omega}|\nabla u|^{2} d x .
$$

Finally

i) If $k \leq 0$, then

$$
-\dot{V}(u) \geq c \int_{\Omega}|\nabla u|^{2} d x, \quad c=a^{2}\left(\frac{1}{\omega_{N}}|\Omega|\right)^{-2 / N}
$$

ii) If $0<k<\frac{a}{2}\left(\frac{1}{\omega_{N}}|\Omega|\right)^{-2 / N}$

it follows from (2.5), (2.6) and (2.8) that

$$
-\dot{V}(u) \geq \delta \int_{\Omega}|\nabla u|^{2} d x
$$

with $\delta=a^{2}\left(\frac{1}{\omega_{N}}|\Omega|\right)^{-2 / N}-2 a k>0$ and we obtain, by the Lemma 2.1, in any case, $i$ ) or $i i)$, the asymptotic stability and the decay $\left\|u\left(t, u_{0}\right)\right\|_{H^{1}} \rightarrow 0$, as $t \rightarrow \infty$, for any $u_{0} \in H_{0}^{1}(\Omega)$. 


\section{Proof of the Theorem 1.5}

Consider the (CGL) problem with $a, k>0, b<0, \alpha, \beta \in \mathbb{R}$ and $\Omega$ a bounded domain of class $C^{2}$. Assume that $0<\sigma \leq \frac{2}{N-2}$ if $N>2,0<\sigma$ if $N=1,2$ and $\alpha / a=\beta / b$. As we pointed out before, for any $u_{0} \in H_{0}^{1}(\Omega)$ there exists a maximal solution of (CGL) defined on $[0, T), T>0$, denoted by $S(t) u_{0} \equiv u\left(t, u_{0}\right)$. Suppose that $\sup _{t>0}\left\|S(t) u_{0}\right\|_{H^{1}} \leq M$ for some $M>0$. Then $\left\{S(t) u_{0}\right\}_{t>0}$ is a relatively compact set in $H_{0}^{1}(\Omega)$ (this is a consequence of the fact that the operator $(a+i \alpha) \Delta$ has compact resolvent (see e.g. [10], pag.57)) and therefore, it follows that the $\omega$ - limit set of $u_{0}$,

$$
\omega\left(u_{0}\right)=\left\{y \in H_{0}^{1}(\Omega): \text { there exists } t_{n} \rightarrow \infty \text { such that } S\left(t_{n}\right) u_{0} \rightarrow y\right\},
$$

is a nonempty, invariant and compact set (see e.g. [4]).

Consider once again the Lyapunov functional

$$
V(u):=\frac{1}{2} \int_{\Omega}\left\{a|\nabla u|^{2}+\frac{2 b}{\sigma+2}|u|^{\sigma+2}-k|u|^{2}\right\} d x
$$

and note (see $(2.4))$ that for any $u \in H_{0}^{1}(\Omega), \dot{V}(u)=0$ if and only if

$$
-\Delta u-\frac{k}{a} u=-\frac{b}{a}|u|^{\sigma} u
$$

Proof of Theorem 1.5. Step 1. First of all we show that there exists a small enough positive number, $\eta>0$, such that do not exists a nontrivial solution $u \in H_{0}^{1}(\Omega)$ of $(3.1)$ with $\|u\|_{H^{1}}=\eta$. Let $u \in H_{0}^{1}(\Omega),\|u\|_{H^{1}}=\eta$ be a solution of (3.1). Since $k / a \in \rho(-\Delta)$ and $|u|^{\sigma} u \in L^{2}(\Omega)$ it follows that $u \in H^{2}(\Omega) \cap H_{0}^{1}(\Omega)$ and

$$
\|u\|_{H^{2}} \leq c\|u\|_{H^{1}}^{\sigma+1} \leq c \eta^{\sigma+1}
$$

which implies $u \equiv 0$ if $\eta$ is small enough.

Step 2. Consider the set

$$
K=\left\{\phi \in H_{0}^{1}(\Omega): V(\phi)<0\right\}
$$

and let be $k / a \in\left(\lambda_{n}, \lambda_{n+1}\right)$ for some $n=1,2, \cdots$, with $\lambda_{n}$ the eigenvalues of $-\Delta$. Then, for any $\varepsilon>0, K \cap U_{\varepsilon}(0) \neq \emptyset$, where $U_{\varepsilon}(0)$ is the $\varepsilon$ - neighbourhood of 0 in $H_{0}^{1}(\Omega)$. To prove that, take $k / a=\lambda_{n}+\delta, 0<\delta<\lambda_{n+1}-\lambda_{n}$ and consider $u_{n}$ the eigenfunction associated to $\lambda_{n}$ with $\left\|u_{n}\right\|_{H^{1}}<\varepsilon$. Since $-\Delta u_{n}=\lambda_{n} u_{n}$ we obtain

$$
\begin{array}{r}
a \int_{\Omega}\left|\nabla u_{n}\right|^{2} d x-k \int_{\Omega}\left|u_{n}\right|^{2} d x=a\left[\int_{\Omega}\left|\nabla u_{n}\right|^{2} d x-\frac{k}{a} \int_{\Omega}\left|u_{n}\right|^{2} d x\right] \\
=a\left[\int_{\Omega}\left|\nabla u_{n}\right|^{2} d x-\lambda_{n} \int_{\Omega}\left|u_{n}\right|^{2} d x-\delta \int_{\Omega}|u|^{2} d x\right]<0
\end{array}
$$

and so $V\left(u_{n}\right)<0$. 
Step 3. Let $U$ be an open neighbourhood of 0 in $H_{0}^{1}(\Omega)$ such that $U \subset$ $\bar{U} \subset U_{\eta}(0)$, where $\eta>0$ was determined before in the step 1 . and let be $\phi \in K \cap U$. We claim that $S(t) \phi \in \partial \bar{U}$ for some $t>0$.

Suppose that we have $\{S(t) \phi\} \subset U$ for all $t>0$. Since $\overline{\{S(t) \phi\}}$ is a compact set and $V(S(t) \phi)$ is a nonincreasing function of $t \geq 0$, it turns out that $l=\lim _{t \rightarrow \infty} V(S(t) \phi)$ exists in $\mathbb{R}$. On the other hand, by the precompacity of $\{S(t) \phi\}_{t \geq 0}$, the $\omega$ - limit set $\omega(\phi)$ is nonempty. Let be $y \in \omega(\phi) \subset U_{\eta}(0)$; it is clear that $V(y)=l$ and, by the invariance of $\omega(\phi), S(t) y \in \omega(\phi)$ and so $V(S(t) y)=l$. It follows that $\dot{V}(y)=0$ and by the step $1 ., y=0$. Therefore, we conclude that $\omega(\phi)=\{0\}$. But, for any $z \in \omega(\phi), z=\lim _{t_{n} \rightarrow \infty} S\left(t_{n}\right) \phi$,

$$
V(z)=\lim _{t_{n} \rightarrow \infty} V\left(S\left(t_{n}\right) \phi\right) \leq V(\phi)<0
$$

which is absurd. We have proved that $S(t) \phi$ must reach $\partial \bar{U}$ for some $t>0$ which means the instability of the equilibrium solution $u \equiv 0$.

\section{Existence and stability of Bound-States of (CGL*)}

Proof of Proposition 1.6. Multiply the equation by $\bar{\phi}$ and integrate:

$$
\omega\|\phi\|_{2}^{2}+\sin \theta\|\nabla \phi\|_{2}^{2}=\sin \gamma\|\phi\|_{\sigma+2}^{\sigma+2}, \quad \cos \gamma\|\nabla \phi\|_{2}^{2}=\cos \gamma\|\phi\|_{\sigma+2}^{\sigma+2} .
$$

Then one has $\omega \cos \theta\|\phi\|_{2}^{2}=\sin (\gamma-\theta)\|\phi\|_{\sigma+2}^{\sigma+2}$. If $\phi \not \equiv 0$, then either $\theta=\pi / 2$ and

$$
\sin (\gamma-\theta)=0
$$

or $\theta<\pi / 2$ and

$$
\sin (\gamma-\theta)>0
$$

From the assumptions on $\gamma$ and $\theta$, both cases are impossible.

We now focus on the proof of Theorem 1.8. Define $H:=\left\{u \in H_{0}^{1}(\Omega)\right.$ : $\left.\Delta u \in L^{2}(\Omega)\right\}$. This space is a real Hilbert space when equipped with the scalar product

$$
(u, v)=\Re \int_{\Omega} u \bar{v}+\Re \int_{\Omega} \Delta u \Delta \bar{v} .
$$

Let $\lambda$ be an eigenvalue of $-\Delta: H \mapsto L^{2}(\Omega)$. Assuming that the corresponding eigenspace is of the form $\mathbb{C} \phi$, we set

$$
H=\mathbb{C} \phi \oplus H_{1}, \quad H_{1}=(\mathbb{C} \phi)^{\perp} .
$$

Lemma 4.1. Suppose that $\Omega$ is a bounded, connected, open subset of $\mathbb{R}^{d}$ such that the correspoding Laplace-Dirichlet operator has a simple eigenvalue $\lambda$. Fix $\theta, \gamma \in \mathbb{R}$ and $\sigma>0$. Then there exist $\mu_{0}>0$ and $C^{1}$ mappings

$$
v:\left(-\mu_{0}, \mu_{0}\right) \mapsto H, \quad \omega, k:\left(-\mu_{0}, \mu_{0}\right) \mapsto \mathbb{R}
$$

such that $v(0)=\phi, \omega(0)=-\lambda \sin \theta, k(0)=\lambda \cos \theta$ and

$$
\Delta v+\mu e^{i(\gamma-\theta)}|v|^{\sigma} v+(k-i \omega) e^{-i \theta} v=0 .
$$


Proof. Define the mapping $F: \mathbb{R} \times H_{1} \times \mathbb{R} \times \mathbb{R} \mapsto L^{2}(\Omega)$ as

$$
F(\mu, \zeta, \omega, k)=\Delta v+\mu e^{i(\gamma-\theta)}|v|^{\sigma} v+(k-i \omega) e^{-i \theta} v, \quad v=\phi+\zeta .
$$

If one sets

$$
k_{0}-i \omega_{0}=\lambda e^{i \theta},
$$

it follows that $F\left(0,0, \omega_{0}, k_{0}\right)=0$. Furthermore, the mapping $(\zeta, \omega, k) \mapsto$ $F(\mu, \zeta, \omega, k)$ is of class $C^{1}$ and

$$
\begin{gathered}
\frac{\partial F}{\partial \zeta}(\mu, \zeta, \omega, k) w=\Delta w+\mu e^{i(\gamma-\theta)}\left(|v|^{\sigma} w+\sigma|v|^{\sigma-2} v \Re(\bar{v} w)\right)+(k-i \omega) e^{-i \theta} w, \\
\frac{\partial F}{\partial \omega}(\mu, \zeta, \omega, k)=-i e^{-i \theta} v, \quad \frac{\partial F}{\partial k}(\mu, \zeta, \omega, k)=e^{-i \theta} v .
\end{gathered}
$$

Now we check that the jacobian

$$
J=\frac{\partial F}{\partial(\zeta, \omega, k)}\left(0,0, \omega_{0}, k_{0}\right): H_{1} \times \mathbb{R} \times \mathbb{R} \mapsto L^{2}(\Omega)
$$

is a bijection. Applying to an element $(w, y, z) \in H_{1} \times \mathbb{R} \times \mathbb{R}$, we have

$$
J(w, y, z)=\Delta w+\lambda w+e^{-i \theta}(z-i y) \phi .
$$

If $J(w, y, z)=0$, then

$$
0=\int J(w, y, z) \bar{\phi}=e^{-i \theta}(z-i y)\|\phi\|_{2}^{2},
$$

which implies $y, z=0$. Thus $-\Delta w=\lambda w$ and so $w$ is an eigenvector with eigenvalue $\lambda$. However, since $w \in H_{1}$, this means that $w=0$. Hence $J$ is injective. On the other hand, given $f \in L^{2}(\Omega)$, write

$$
f=-e^{-i \theta}(\tilde{z}-i \tilde{y}) \phi+\psi, \quad \int_{\Omega} \psi \bar{\phi}=0 .
$$

The orthogonality condition implies that there exists $\tilde{w} \in H_{1}$ such that $\Delta \tilde{w}+\lambda \tilde{w}=\psi$. Then

$$
J(\tilde{w}, \tilde{y}, \tilde{z})=f
$$

which shows that $J$ is surjective.

With the above considerations, one may apply the Implicit Function Theorem [15, Theorem 4.B] and the proof is finished.

Proof of Theorem 1.8. Consider the mappings $v, \omega, k$ from the previous theorem and the mapping $F$ as in (4.1). Then

$$
F(\mu, \zeta(\mu), \omega(\mu), k(\mu))=0, \quad \mu \in\left(-\mu_{0}, \mu_{0}\right) .
$$

Differentiating with respect to $\mu$ at $\mu=0$, we obtain

$$
e^{i(\gamma-\theta)}|\phi|^{\sigma} \phi+\Delta w+\lambda w+e^{-i \theta}\left(\frac{\partial k}{\partial \mu}-i \frac{\partial \omega}{\partial \mu}\right) \phi=0, \quad w=\frac{\partial v}{\partial \mu} .
$$

Multiplying by $\bar{\phi}$ and integrating over $\Omega$, we arrive at

$$
\frac{\partial k}{\partial \mu}=-\cos \gamma \frac{\|\phi\|_{\sigma+2}^{\sigma+2}}{\|\phi\|_{2}^{2}}<0, \quad \frac{\partial \omega}{\partial \mu}=\sin \gamma \frac{\|\phi\|_{\sigma+2}^{\sigma+2}}{\|\phi\|_{2}^{2}} .
$$


Thus the mapping $\mu \mapsto k(\mu)$ is locally invertible at 0 , which implies that one may write $\mu=\mu(k), v=v(k)$ and $\omega=\omega(k)$, for $|k-\lambda \cos \theta|<\epsilon$. Finally, if $\lambda \cos \theta-k>0$, then $\mu(k)>0$ and so $u(k)=\mu(k)^{\frac{1}{\sigma}} v(k)$ satisfies (B-S).

Proof of Theorem 1.10. We start with the uniqueness statement. We write the equation as a system of ODE's and linearize around the trivial solution. One then checks that the linear system has two eigenvalues $\pm \lambda$, with $\Re \lambda>0$ and that each eigenvalue has a two-dimensional eigenspace. It follows that stable and unstable manifolds $(\mathcal{S}$ and $\mathcal{U}$ ) for the full equation $(\mathrm{B}-\mathrm{S})$ have dimension two.

Given a bound-state $\phi \in \mathcal{U} \cap \mathcal{S}$, define

$$
\mathcal{U}_{0}:=\left\{e^{i \rho}\left(\phi(x), \phi^{\prime}(x)\right): \rho \in \mathbb{T}, x \in \mathbb{R}\right\} .
$$

We claim that, if for some $\left(\rho_{0}, x_{0}\right) \neq\left(\rho_{1}, x_{1}\right)$,

$$
e^{i \rho_{0}}\left(\phi\left(x_{0}\right), \phi^{\prime}\left(x_{0}\right)\right)=e^{i \rho_{1}}\left(\phi\left(x_{1}\right), \phi^{\prime}\left(x_{1}\right)\right),
$$

then $\phi$ would not go to 0 as $t \rightarrow+\infty$. Indeed, either $x_{1}=x_{0}$ (which implies the contradiction $\rho_{1}=\rho_{2}$ ) or $x_{1} \neq x_{0}$. If $x_{1}>x_{0}$, then the gauge and translation invariances of the equation imply that, for $\delta=x_{1}-x_{0}$,

$$
\left(\phi\left(x_{0}+n \delta\right), \phi^{\prime}\left(x_{0}+n \delta\right)\right)=e^{i n\left(\rho_{0}-\rho_{1}\right)}\left(\phi\left(x_{0}\right), \phi^{\prime}\left(x_{0}\right)\right), \quad n \in \mathbb{N}
$$

which is again a contradiction when $n \rightarrow \infty$. Hence $\mathcal{U}_{0}$ is a two-dimensional submanifold of $\mathcal{U}$.

Next, we show that $\mathcal{U}_{0}$ is closed in $\mathcal{U}$ : given $(y, z) \in \mathcal{U} \cap \overline{\mathcal{U}_{0}}$, then

$$
e^{i \rho_{n}}\left(\phi\left(x_{n}\right), \phi^{\prime}\left(x_{n}\right)\right) \rightarrow(y, z), \quad\left(\rho_{n}, x_{n}\right) \in \mathbb{T} \times \mathbb{R} .
$$

If $\left(\rho_{n}, x_{n}\right), n \in \mathbb{N}$, remains in a bounded set, then, up to a subsequence, $\left(\rho_{n}, x_{n}\right) \rightarrow\left(\rho_{0}, x_{0}\right)$ and so

$$
(y, z)=e^{i \rho_{0}}\left(\phi\left(x_{0}\right), \phi^{\prime}\left(x_{0}\right)\right) \in \mathcal{U}_{0} .
$$

If not, then there exists a subsequence $\left(x_{n_{j}}\right)_{j \in \mathbb{N}}$ such that $x_{n_{j}} \rightarrow \pm \infty$. But then $(y, z)=(0,0) \notin \mathcal{U}$, which is absurd. We conclude that $\mathcal{U}_{0}$ is closed in $\mathcal{U}$

From the invariance of domains, $\mathcal{U}_{0}$ is open in $\mathcal{U}$. Since $\mathcal{U}$ is connected, $\mathcal{U}_{0}=\mathcal{U}$ and so, up to gauge rotations, there can only be one solution on the unstable manifold. Since any solution of (B-S) must lie on $\mathcal{U}$, one obtains uniqueness of bound-states.

Now we focus on the existence statement. We look for solutions $\phi \in$ $H^{1}(\mathbb{R})$ of $(\mathrm{B}-\mathrm{S})$ or, in an equivalent way,

$$
\phi^{\prime \prime}=\omega e^{i \tilde{\theta}} \phi-e^{i \tilde{\gamma}}|\phi|^{\sigma} \phi+i k e^{i \tilde{\theta}} \phi
$$

with $\tilde{\theta}=\pi / 2-\theta, \tilde{\gamma}=\gamma-\theta$.

Let us search a solution of the equation (4.2), $\phi \in H^{1}(\mathbb{R})$, of the form

$$
\phi=\psi \exp (i d \ln \psi)
$$


where $d \in \mathbb{R}$ and $\psi>0$ is the unique solution of the stationary Schrödinger equation

$$
\psi^{\prime \prime}=\epsilon \psi-\eta \psi^{\sigma+1}, \quad \epsilon, \eta>0 .
$$

First, one has

$$
\phi^{\prime \prime}(x)=\left[\psi^{\prime \prime}(x)(1+i d)+i d(1+i d) \frac{\psi^{\prime}(x)^{2}}{\psi(x)}\right] \exp (i d \ln \psi(x)) .
$$

Next, we note that if $\psi$ is a solution of (4.4), then a direct integration of the equation yields

$$
\frac{\left(\psi^{\prime}\right)^{2}}{\psi}=\epsilon \psi-\frac{2 \eta}{\sigma+2} \psi^{\sigma+1} .
$$

It follows from (4.2) that

$$
\begin{aligned}
& \psi^{\prime \prime}-d^{2} \frac{\left(\psi^{\prime}\right)^{2}}{a}=\omega \cos \tilde{\theta} \psi-k \sin \tilde{\theta} \psi-\cos \tilde{\gamma} \psi^{\sigma+1}, \\
& d \psi^{\prime \prime}+d \frac{\left(\psi^{\prime}\right)^{2}}{\psi}=\omega \sin \tilde{\theta} \psi+k \cos \tilde{\theta} \psi-\sin \tilde{\gamma} \psi^{\sigma+1}
\end{aligned}
$$

and so

$$
\begin{aligned}
& \left(1+d^{2}\right) \psi^{\prime \prime}=[\omega(d \sin \tilde{\theta}+\cos \tilde{\theta})+k(d \cos \tilde{\theta}-\sin \tilde{\theta})] \psi \\
& -(d \sin \tilde{\gamma}+\cos \tilde{\gamma}) \psi^{\sigma+1}, \\
& \left(1+d^{2}\right) \frac{\left(\psi^{\prime}\right)^{2}}{\psi}=\left[\omega\left(\frac{\sin \tilde{\theta}}{d}-\cos \tilde{\theta}\right) \psi+k\left(\frac{\cos \tilde{\theta}}{d}+\sin \tilde{\theta}\right) \psi\right. \\
& \left.-\left(\frac{\sin \tilde{\gamma}}{d}-\cos \tilde{\gamma}\right) \psi^{\sigma+1}\right] .
\end{aligned}
$$

Hence, writing

$$
\epsilon=\frac{\omega(d \sin \tilde{\theta}+\cos \tilde{\theta})+k(d \cos \tilde{\theta}-\sin \tilde{\theta})}{1+d^{2}}
$$

and

$$
\eta=\frac{d \sin \tilde{\gamma}+\cos \tilde{\gamma}}{1+d^{2}}
$$

we require that

$$
\omega\left(\frac{\sin \tilde{\theta}}{d}-\cos \tilde{\theta}\right)+k\left(\frac{\cos \tilde{\theta}}{d}+\sin \tilde{\theta}\right)=\omega(d \sin \tilde{\theta}+\cos \tilde{\theta})+k(d \cos \tilde{\theta}-\sin \tilde{\theta})
$$

and

$$
\frac{\sin \tilde{\gamma}}{d}-\cos \tilde{\gamma}=\frac{2}{\sigma+2}(d \sin \tilde{\gamma}+\cos \tilde{\gamma})
$$


From (4.9) we derive

$$
d=\frac{k \sin \tilde{\theta}-\omega \cos \tilde{\theta} \pm \sqrt{\omega^{2}+k^{2}}}{\omega \sin \tilde{\theta}+k \cos \tilde{\theta}}=: d_{ \pm}
$$

and so

$$
\epsilon= \pm \sqrt{\omega^{2}+k^{2}}
$$

However, if $d=d_{-}$then $\psi$ would be a bound state of a nonlinear Schrödinger equation with negative frequency, $\epsilon$, which does not exists (see [1]). Thus, we must have $d=d_{+}$. Finally, the definition of $\gamma$ (cf. (1.5)) is equivalent to (4.10) and $\eta>0$.

Finally, we turn our attention to the proof of Theorem 1.13. We start to notice that a bound-state $\phi \in H^{1}(\mathbb{R})$ of $\left(\mathrm{CGL}^{*}\right)$ is an equilibrium point of the equation

$$
u_{t}=e^{i \theta} u^{\prime \prime}+e^{i \gamma}|u|^{\sigma} u-i \omega u+k u .
$$

Linearizing around $\phi$, we obtain

$$
u_{t}=e^{i \theta} u^{\prime \prime}+e^{i \gamma}|\phi|^{\sigma} u+\sigma e^{i \gamma}|\phi|^{\sigma-2} \phi \Re(\bar{\phi} u)-i \omega u+k u \equiv-L u .
$$

If the spectrum $\sigma(L)$ is in $\{\Re \lambda>\eta\}$ for some $\eta>0$, it is well known that the equilibrium point $\phi$ of (4.13) is asymptotically stable and thus we need some information about the spectrum of the operator $L$.

Definition 4.2. Let $L$ be a linear operator in a Banach space. We call a normal point any element of the resolvent set or an isolated eigenvalue of $L$ of finite multiplicity. The set of the normal points of $L$ is represented by $\tilde{\rho}(L)$. We define the essencial spectrum of $L$ as the set $\sigma_{e}(L):=\mathbb{C} \backslash \tilde{\rho}(L)$.

Consider now the operator $L$ given in (4.13), $L=M+N$ with

$$
M u=-e^{i \theta} u^{\prime \prime}+i \omega u-k u, \quad D(M)=H^{2}(\mathbb{R})
$$

and

$$
N u=-e^{i \gamma}|\phi|^{\sigma} u-\sigma e^{i \gamma}|\phi|^{\sigma-2} \phi \Re(\bar{\phi} u)
$$

Notice that $N$ is a linear bounded operator on $L^{2}(\mathbb{R})$ ( since $\phi \in H^{1}(\mathbb{R}) \subset$ $\left.L^{\infty}(\mathbb{R})\right)$. The next lemma determines, in a way, the location of the essential spectrum, $\sigma_{e}(L)$.

Lemma 4.3. 1 . The operator $N\left(\lambda_{0}-M\right)^{-1}$ is compact for some $\lambda_{0} \in \mathbb{R}$.

2. Let $k<0$ and consider the half-plane $\Sigma=\{\Re \lambda<-k\} \subset \rho(M)$. Then, either $\Sigma$ consists of normal points of $M+N$ or consists entirely of eigenvalues of $M+N$, and so $\sigma_{e}(L) \subset\{\Re \lambda \geq-k\}$.

Proof. 1. Take a sequence $\chi_{j} \in C_{0}^{\infty}(\mathbb{R})$ such that

$$
\chi_{j}(x)=1, x \in[-j, j], \operatorname{supp} \chi_{j} \subset[-(j+1), j+1]
$$

and consider the operators

$$
N_{j} u=-e^{i \gamma}\left|\phi_{j}\right|^{\sigma} u-\sigma e^{i \gamma}\left|\phi_{j}\right|^{\sigma-2} \phi \Re\left(\overline{\phi_{j}} u\right)
$$


where $\phi_{j}=\chi_{j} \phi$. For $\lambda_{0} \in \Sigma,\left(\lambda_{0}-M\right)^{-1}: L^{2}(\mathbb{R}) \rightarrow H^{2}(\mathbb{R})$ and we see that, for any $j \in \mathbb{N}, N_{j}\left(\lambda_{0}-M\right)^{-1}$ is a compact operator. On the other hand

$$
N_{j}\left(\lambda_{0}-M\right)^{-1} \rightarrow N\left(\lambda_{0}-M\right)^{-1}(j \rightarrow \infty)
$$

in the operator norm of $\mathcal{L}\left(L^{2}(\mathbb{R})\right)$. Since the space of compact operators is closed in the $\mathcal{L}\left(L^{2}(\mathbb{R})\right)$ space, it follows that $N\left(\lambda_{0}-M\right)^{-1}$ is compact.

2. We can now use [8, Lemma 5.2] to conclude that the connected set $\Sigma \subset \rho(M)$ consists entirely of normal points of $L=M+N$ or entirely of eigenvalues of $L$.

Proof of the Theorem 1.13. Let $\phi=\psi \exp (i d \ln \psi)$ be the bound-state built in Theorem 1.10. Then $\psi$ satisfies

$$
\psi^{\prime \prime}=\epsilon \psi-\eta \psi^{\sigma+1},
$$

where $\epsilon$ and $\eta$ are given by (4.7), (4.8). As a consequence (see [1], pag. 260),

$$
\|\phi\|_{L^{\infty}(\mathbb{R})}=\|\psi\|_{L^{\infty}(\mathbb{R})}=|\psi(0)|=\left[\epsilon\left(\frac{\sigma+2}{2}\right)\right]^{\sigma / \epsilon} \eta^{\sigma} .
$$

We have already seen (Lemma 4.3) that the essential spectum $\sigma_{e}(L)$ lies in $\{\Re \lambda>-k\}$. We need only to determine the location of the eigenvalues of $L=M+N$. Since $\|N u\|_{L^{2}(\mathbb{R})} \leq(1+\sigma)\|\phi\|_{L^{\infty}(\mathbb{R})}^{\sigma}\|u\|_{L^{2}(\mathbb{R})}$, with $\phi$ an $H^{1}$ solution of (4.2), any eigenvalue $\lambda$ of $L$ verifies

$$
\Re \lambda \geq-k-(1+\sigma)\|\phi\|_{L^{\infty}(\mathbb{R})}^{\sigma}
$$

and, therefore, one has the asymptotic stability of the bound-state $e^{i t} \phi$ if

$$
(1+\sigma)\|\phi\|_{L^{\infty}(\mathbb{R})}^{\sigma}<-k .
$$

Noticing that, when $k \rightarrow-\infty$,

$$
\epsilon \sim-\frac{k(1+\cos \theta)}{2}>0 \text { and } \eta \text { bounded, }
$$

condition (4.14) is verified for sufficiently large $-k$.

\section{ACKNOWLEDGEMENTS}

The authors were partially supported by Fundação para a Ciência e Tecnologia, through the grant UID/MAT/04561/2013.

\section{REFERENCES}

[1] Cazenave, T., Semilinear Schrödinger Equations, Courant Lecture Notes in Mathematics, 10. New York University, American Mathematical Society, Providence, R.I., 2003.

[2] Cazenave, T., Dickstein, F. and Weissler, F., Finite time blowup for a complex Ginzburg-Landau equation, SIAM J. Math. Anal., 45 (2013), pp. 244-266.

[3] Cazenave, T., Dickstein, F. and Weissler, F., Standing waves of the complex GinzburgLandau equation, Nonlinear Anal., 103 (2014), pp. 26-32.

[4] Cazenave, T. and Haraux, A., An introduction to semilinear evolution equations, Oxford Lectures Series in Mathematics and its Applications, Oxford University Press, New York, 1998. 
[5] Cipolatti, R., Dickstein, F and Puel, J-P., Existence of standing waves for the complex Ginzburg-Landau equation, J. Math. Anal. Appl., 422 (2015), pp. 579-593.

[6] Ginibre, J. and Velo, G., The Cauchy problem in local spaces for the complex GinzburgLandau equation I : Compactness methods, Phys. D, 95 (1996), pp. 191-228.

[7] Ginibre, J. and Velo, G., The Cauchy problem in local spaces for the complex GinzburgLandau equation II : Contraction methods, Comm. Math. Phys., 187 (1997), pp. 45-79.

[8] Gohberg, I.G. and Krein, M.G., Introduction to the Theory of Linear Nonselfadjoint Operators, Transl. Math. Monog., Vol. 18 (1969) Am. Math. Soc., Providence, R.I.

[9] Hale, J.K., Dynamical systems and stability, J. Math. Anal. Appl., 26 (1969), pp. 39-59.

[10] Henry, D., Geometric theory of semilinear parabolic equations, Lecture Notes in Math., 840, Springer, New York, 1981.

[11] Levermore, C.D. and Oliver, M., The complex Ginzburg-Landau equation as a model problem, Lectures in Appl. Math. 31, AMS, Providence, R.I., 1996, pp. 141-190.

[12] Masmoudi N. and Zaag H., Blow-up profile for the complex Ginzburg-Landau equation, J. Funct. Anal., 255 (2008), pp. 1613-1666.

[13] Okazawa, N. and Yokota, T., Subdifferential operator approach to strong wellposedness of the complex Ginzburg-Landau equation, Discrete Contin. Dyn. Syst., 28 (2010), pp. 311-341.

[14] Pazy, A., Semi-groups of linear operators and applications to partial differential equations, Springer, Berlin, 1983.

[15] Zeidler, E., Nonlinear functional analysis and its applications. I, Springer-Verlag, New York, 1986.

SimÃo CORREIA

CMAF-CIO, Universidade de Lisboa

Edifício C6, Campo Grande

1749-016 Lisboa, Portugal

sfcorreia@fc.ul.pt

Mário Figueira

CMAF-CIO, Universidade de Lisboa

Edifício C6, Campo Grande

1749-016 Lisboa, Portugal

msfigueira@fc.ul.pt 